\title{
EFFECTS OF CULTURE MEDIA ON YIELD, GROWTH, PHOTOSYNTHETIC CAPACITY AND ANTIOXIDANT ENZYME OF DIFFERENT TOMATO (SOLANUM LYCOPERSICUM L.) VARIETIES
}

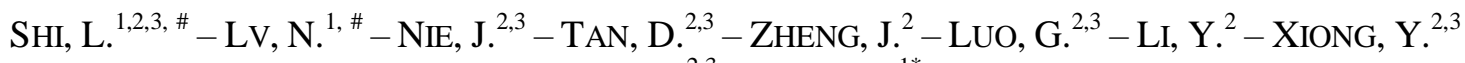 \\ $-\mathrm{XIE}, \mathrm{Y}^{2,3}-\mathrm{WANG}, \mathrm{J}^{{ }^{*}}$ \\ ${ }^{1}$ Key Laboratory of Agroenvironment in Tropic, Ministry of Agriculture, South China \\ Agricultural University, 510642 Guangzhou, China \\ ${ }^{2}$ Agro-innovative Demonstration Base, Guangdong Academy of Agricultural Sciences \\ 510642 Guangzhou, China \\ ${ }^{3}$ Key Laboratory of Urban Agriculture in South China, Ministry of Agriculture \\ 510640 Guangzhou, China \\ *Corresponding author \\ e-mail:wangjw@scau.edu.cn \\ ${ }^{\#}$ These authors contributed equally to this work. \\ (Received $15^{\text {th }}$ Jun 2018; accepted 22 ${ }^{\text {nd }}$ Aug 2018)
}

\begin{abstract}
Culture media affects the yield and quality of tomato. Knowledge about the tomato yield formation in response to culture media is still needed. The purpose of this study was to investigate the effect of culture media on yield, growth, photosynthetic capacity and antioxidant enzyme of different tomato varieties. A greenhouse experiment was conducted at the Baiyun Experimental Base of Guangdong Academy of Agricultural Sciences, Guangdong, China, three culture media treatments viz. soil culture cultivation treatment, substrate cultivation treatment and hydroponics cultivation treatment were applied. Four represented tomato varieties viz. Xinxing 101, Jinbaolai, Kaidi and Xiariyangguang were used as materials. The result showed that, the substrate cultivation and hydroponics produced higher yield than soil culture treatment due to higher dry weight accumulation, fruit weight, and net photosynthetic rate at seedling stage and mature stage. The yield showed significant correlation with individual fruit weight $\left(\mathrm{R}^{2}=0.8205, P<0.01\right)$, net photosynthetic rate in leaves at seedling stage $\left(\mathrm{R}^{2}=0.7189, P<0.05\right)$. The SPAD value and antienzyme activity vary among different planting patterns, cultivars and growth stages. Further study to observe the quality of tomato fruits and nutrition of the tomato plant under different culture media is needed.
\end{abstract}

Keywords: antioxidant capacity, culture cultivation, plant growth, photosynthetic ability, tomato, yield formation

\section{Introduction}

Tomato is an important vegetable crop worldwide which ranks next to spuds in terms of the planted area whilst ranks first as a processing crop (Mehdizadeh et al., 2013). It is susceptible to pest and disease while water management and nutrient supply could significantly affect tomato's health (Salokhe et al., 2005). However, this problem could be solved in greenhouse condition which has been known as a protected cultivation (Pires et al., 2011). Recently tomato is suitable for facility cultivation for its high yield and quality requirement and precise management for pest and disease control (Xie et al., 2017). Greenhouse farming, one of the famous agriculture systems, is widely used to create and maintain a stable and suitable environment to optimize the crop production in 
order to maximum profits (Abou-Hadid et al., 1994). For facility cultivation of tomato, the culture media is one of the most important key factors that affecting the yield and quality of tomato for the cultivated culture media are different in physicochemical properties such as water-retaining property, nutrient preserving capability, heat retaining property, gas permeability and so on (Liu et al., 2006).

A previous study revealed that different nitrogen forms in media culture had significant effects on tomato's photosynthetic rate, plant growth, root vigor and leaf water content (Yang, 2003). Previous report demonstrated that silicate application in culture media affected tomato fruits and silicon content (Lee et al., 2002). Furthermore, supplementing the nutrient solution with $\mathrm{NaCl}$ was able to decrease the yield of tomato significantly (Korkmaz et al., 2016). Thus, it is commonly recommended to select appropriate cultivated culture media for the tomato growth and to improve plant establishment and enhance yield.

Photosynthesis is the basis of crop yield and has a significant correlation with both the yield and fruit quality of tomato (Xu et al., 2001). Regarding to photosynthetic traits, different growth culture media will have effect on net photosynthetic rate, transpiration rate, stomatal conductance and Intercellular carbon dioxide. A previous report showed that culture on sugar medium enhances photosynthetic capacity and high light resistance of plantlets grown in vitro (Tichá et al., 2010). The study of Chen (Chen et al., 2009) demonstrated that tomato assimilation products were enhanced in organic substrates compared with the soil control while the photosynthetic rate and intracellular $\mathrm{CO}_{2}$ concentration were higher in organic substrate cultivation. Ghoreishy et al. (2018) found that tomato grown in culture media which using composted wheat residue had better fruit yield, chlorophyll, and vitamin C contents. In addition, Hydroponics is also a modern agriculture technique which uses nutrient solution for crop production and a report demonstrated that hydroponic greenhouse system is only system which could meet the food requirements of our needs (Khan, 2018).

However, effect of culture media on yield, growth, photosynthetic capacity and antioxidant enzyme of different tomato varieties is still unknown. Here, we studied the effect of culture media viz. soil culture cultivation treatment, substrate cultivation treatment and hydroponics cultivation treatment on yield, growth, photosynthetic capacity and antioxidant enzyme of four represented tomato varieties viz. Xinxing 101, Jinbaolai, Kaidi and Xiariyangguang.

\section{Materials and methods}

\section{Tomato plant growth condition}

A greenhouse experiment was conducted from 2016 to 2017 at the Baiyun Experimental Base of Guangdong Academy of Agricultural Sciences, Guangdong, China. Four popular tomato cultivars were used in this study, two of them were large fruit tomato cultivar (Xinxing 101 and Jinbaolai), the other two were small fruit tomato (Kaidi and Xiariyangguang), these four cultivars can be planted in spring, autumn or winter and is provided by the Modern Demonstration Garden of Guangdong Academy of agricultural Sciences. The seeds of the tomato cultivar were sown at March $15^{\text {th }}$ and the seedlings were transplanted at April $22^{\text {nd }}$. Two seedlings were transplanted to each foam box, the foam box (Length $\times$ Width $\times$ Height $=59.5 \mathrm{~cm} \times 36 \mathrm{~cm} \times 45 \mathrm{~cm}$ ) purchased from Guangzhou Xinyinfa Foam Products co. LTD. The climatic conditions 
applied during plant growth period was $30 \pm 5{ }^{\circ} \mathrm{C}$ in air temperature and $70 \pm 20 \%$ in air humidity.

\section{Experimental design}

The tomato cultivars were growing under three culture media: I) Soil culture filled the foam box with $80 \mathrm{~kg}$ soil. II) Substrate cultivation, filled the foam box with $20 \mathrm{~kg}$ substrate (vermiculite: pearlite: ceramsite: turfy earth $=1: 1: 1.5: 3$ ). III) Hydroponics, filled the foam box with $85 \mathrm{~L}$ nutrient solution, the nutrient solution containing $\mathrm{Ca}\left(\mathrm{NO}_{3}\right)_{2} \cdot 4 \mathrm{H}_{2} \mathrm{O} 590 \mathrm{mg} \cdot \mathrm{L}^{-1}, \mathrm{KNO}_{3} 404 \mathrm{mg} \cdot \mathrm{L}^{-1}, \mathrm{KH}_{2} \mathrm{PO}_{4} 136 \mathrm{mg} \cdot \mathrm{L}^{-1}, \mathrm{MgSO}_{4} \cdot 7 \mathrm{H}_{2} \mathrm{O}$ $246 \mathrm{mg} \cdot \mathrm{L}^{-1}, \mathrm{MnSO}_{4} \cdot \mathrm{H}_{2} \mathrm{O} 2.13 \mathrm{mg} \cdot \mathrm{L}^{-1}, \mathrm{H}_{3} \mathrm{BO}_{3} 2.86 \mathrm{mg} \cdot \mathrm{L}^{-1}, \mathrm{ZnSO}_{4} \cdot 7 \mathrm{H}_{2} \mathrm{O} 0.22 \mathrm{mg} \cdot \mathrm{L}^{-1}$, $\mathrm{CuSO}_{4} \cdot 5 \mathrm{H}_{2} \mathrm{O} 0.08 \mathrm{mg} \cdot \mathrm{L}^{-1}$, and $\left(\mathrm{NH}_{4}\right)_{6} \mathrm{Mo}_{7} \mathrm{O}_{24} \cdot 4 \mathrm{H}_{2} \mathrm{O} 0.02 \mathrm{mg} \cdot \mathrm{L}^{-1}$ (Lv et al., 2010). For the soil culture and substrate cultivation treatment, each foam box was irrigated with $400 \mathrm{ml}$ of the nutrient solution every two days. For the hydroponics treatment, the nutrient solution kept constant volume.

\section{Sampling and measurements}

Fruit weight and yield of tomato

The mature fruit was harvest during November 2016 to February 2017, the fruit numbers per was calculated, the individual fruit weight and yield was investigated.

\section{Dry weight of tomato plants}

At harvest stage, three represented plants in each treatment were harvested, the plants were separated into stem and leaves, the different plant parts were oved- dried at $80{ }^{\circ} \mathrm{C}$ to constant weight for measurement of dry weight of tomato plant.

\section{SPAD value and net photosynthetic rate $(\mathrm{Pn})$ in leaves}

The SPAD value and net photosynthetic rate $(\mathrm{Pn})$ in leaves was measured at seedling stage (2016-08-26), flowering stage (2016-10-21) and mature stage (2017-01-20). Three represented plants in each treatment were selected for the measurement of SPAD value and Pn. For SPAD value, the SPAD value was detected by using the SPAD-502 reading meter (KONICA MINOLTA, Japan). The Pn $\left(\mu \mathrm{mol} \cdot \mathrm{m}^{-2} \cdot \mathrm{s}^{-1}\right)$ was measured using LI6400 (LI-COR, USA). Measurement was conducted in the morning (9:30 - 11:30) in each stage.

\section{Superoxide dismutase (SOD), peroxidase (POD), catalase (CAT) activity in leaves}

The fifth leaf from the top of the seven tomato plants were sampled for the measurement of antienzyme activity. The leaves were immediately put in liquid nitrogen and stored at $-80{ }^{\circ} \mathrm{C}$ (for biochemical assays).

Fresh leaf samples were crushed in liquid-nitrogen and homogenized in $3 \mathrm{ml}$ of $50 \mathrm{mM}$ sodium phosphate buffer $(\mathrm{pH} 7.8)$ and the homogenates were centrifuged at $10,000 \mathrm{~g}$ for $15 \mathrm{~min}$ at $4{ }^{\circ} \mathrm{C}$ and the aliquot of the supernatant was used to investigate the enzymatic activities. The SOD activity was measured by detecting the inhibition of photochemical reduction due to nitro blue tetrazolium (NBT) (Foyer, 1987). The POD activity was measured by using guaiacol method (Foyer, 1987). The CAT activity was investigated according to the procedures of Foyer (1987) by recording the decomposition of $\mathrm{H}_{2} \mathrm{O}_{2}$ at $\mathrm{A} 240$. 


\section{Statistical analysis}

Pots were arranged in randomized complete block design (RCBD), the data were analyzed by using a statistical software (SPSS 13.0), the mean value among the different culture media treatments within one variety was separated by LSD method at $P<0.05$ level. Correlation analyses among yield and other parameters were computed and represented by using Excel 2010.

\section{Result}

\section{Yield of different tomato varieties}

Compared to control (soil culture treatment), the hydroponics treatment increased fruit number per plant for all the varieties, while the substrate cultivation increased fruit number per plant in Xinxing101, Jinbaolai, and Kaidi. The substrate cultivation and hydroponics treatment remained statistically similar in individual fruit weight in Xinxing 101, whilst significant increase in Jinbaolai was detected as compared with control (soil culture treatment). In addition, the hydroponics treatment dramatically increased individual fruit weight in Kaidi and Xiariyangguang, but substrate cultivation did not differ from soil culture condiction in individual fruit weight. The yield of tomato showed substantial changes in Xinxing101, Jinbaolai and Kaidi with an increase of 64.87, 77.61, and 48.59\%, respectively for substrate cultivation treatment as compared with soil culture treatment. Moreover, compared to control, all tomato cultivars showed significant improvement in hydroponic treatment, with a percentage increase of 107.41, 58.28, 73.19, and 120.29\% in Xinxing101, Jinbaolai, Kaidi, and Xiariyangguang, respectively (Table 1).

Table 1. Effect of culture media on yield of different tomato varieties

\begin{tabular}{|c|c|c|c|c|c|}
\hline Varieties & Culture media & $\begin{array}{c}\text { Fruit } \\
\text { number } \\
\text { per plant }\end{array}$ & $\begin{array}{c}\text { Individual } \\
\text { fruit weight } \\
\left(\mathrm{g} \cdot \text { fruit }^{-1}\right)\end{array}$ & $\begin{array}{c}\text { Yield } \\
\left(\text { g plant }^{-1}\right)\end{array}$ & $\begin{array}{l}\text { Yield change } \\
\text { relative to soil } \\
\text { culture (\%) }\end{array}$ \\
\hline \multirow{3}{*}{ Xinxing101 } & Soil culture & 199 & $5.02 \pm 0.05 \mathrm{ab}$ & $1000.68 \pm 83.94 b$ & - \\
\hline & Substrate cultivation & 307 & $5.38 \pm 0.10 \mathrm{a}$ & $1649.83 \pm 111.81 \mathrm{a}$ & 64.87 \\
\hline & Hydroponics & 432 & $4.81 \pm 0.19 b$ & $2076.02 \pm 347.04 \mathrm{a}$ & 107.41 \\
\hline \multirow{3}{*}{ Jinbaolai } & Soil culture & 179 & $4.86 \pm 0.02 \mathrm{c}$ & $870.90 \pm 82.66 \mathrm{~b}$ & - \\
\hline & Substrate cultivation & 282 & $5.48 \pm 0.15 b$ & $1546.66 \pm 128.18 \mathrm{a}$ & 77.61 \\
\hline & Hydroponics & 213 & $6.51 \pm 0.16 \mathrm{a}$ & $1378.31 \pm 182.97 \mathrm{a}$ & 58.28 \\
\hline \multirow{3}{*}{ Kaidi } & Soil culture & 270 & $1.53 \pm 0.03 b$ & $413.64 \pm 39.85 c$ & - \\
\hline & Substrate cultivation & 401 & $1.53 \pm 0.02 b$ & $613.78 \pm 20.63 b$ & 48.59 \\
\hline & Hydroponics & 302 & $2.37 \pm 0.06 \mathrm{a}$ & $715.76 \pm 39.96 a$ & 73.19 \\
\hline \multirow{3}{*}{ Xiariyangguang } & Soil culture & 245 & $1.38 \pm 0.00 \mathrm{~b}$ & $338.59 \pm 45.57 \mathrm{~b}$ & - \\
\hline & Substrate cultivation & 237 & $1.56 \pm 0.02 b$ & $369.27 \pm 38.91 \mathrm{~b}$ & 9.11 \\
\hline & Hydroponics & 351 & $2.12 \pm 0.11 \mathrm{a}$ & $745.06 \pm 139.79 a$ & 120.20 \\
\hline
\end{tabular}

Different lowercase letters within one variety represent significant difference at $P<0.05$ level

\section{Dry weight of different tomato varieties}

In response to different culture media, the stem dry weight and leaf dry weight in Xinxing101, Kaidi, and Xiariyangguang was not affected by culture media. The stem 
dry weight in Jinbaolai significantly differed among culture media. The substrate cultivation $(63.33 \%)$ and hydroponics treatment $(128.88 \%)$ recorded significant higher stem dry weight than the soil culture treatment. Hydroponics treatment induced a significant improvement in leaf dry weight by $97.89 \%$ (Table 2).

Table 2. Effect of culture media on dry weight of different tomato varieties

\begin{tabular}{c|c|c|c|c|c}
\hline Varieties & Culture media & $\begin{array}{c}\text { Stem dry } \\
\text { weight (g) }\end{array}$ & $\begin{array}{c}\text { Stem dry } \\
\text { weight change } \\
\text { relative to soil } \\
\text { culture (\%) }\end{array}$ & $\begin{array}{c}\text { Leaf dry } \\
\text { weight (g) }\end{array}$ & $\begin{array}{c}\text { Leaf dry } \\
\text { weight change } \\
\text { relative to soil } \\
\text { culture (\%) }\end{array}$ \\
\hline \multirow{3}{*}{ Xinxing101 } & Soil culture & $23.03 \pm 1.97 \mathrm{a}$ & & $36.78 \pm 7.89 \mathrm{a}$ & \\
& Substrate cultivation & $32.89 \pm 5.63 \mathrm{a}$ & 42.81 & $38.05 \pm 8.67 \mathrm{a}$ & 3.45 \\
& Hydroponics & $31.05 \pm 2.36 \mathrm{a}$ & 34.80 & $37.44 \pm 4.87 \mathrm{a}$ & 1.80 \\
\hline \multirow{3}{*}{ Jinbaolai } & Soil culture & $18.19 \pm 1.63 \mathrm{c}$ & & $22.71 \pm 4.15 \mathrm{~b}$ & \\
& Substrate cultivation & $29.71 \pm 1.41 \mathrm{~b}$ & 63.35 & $25.46 \pm 2.16 \mathrm{~b}$ & 12.09 \\
& Hydroponics & $41.27 \pm 5.31 \mathrm{a}$ & 126.90 & $44.94 \pm 2.30 \mathrm{a}$ & 97.87 \\
\hline \multirow{3}{*}{ Kaidi } & Soil culture & $30.72 \pm 2.43 \mathrm{a}$ & & $33.83 \pm 3.31 \mathrm{a}$ & \\
& Substrate cultivation & $33.50 \pm 3.80 \mathrm{a}$ & 9.07 & $22.24 \pm 1.37 \mathrm{a}$ & -34.27 \\
& Hydroponics & $37.34 \pm 7.88 \mathrm{a}$ & 21.56 & $31.43 \pm 10.02 \mathrm{a}$ & -7.08 \\
\hline \multirow{2}{*}{ Xiariyangguang } & Soil culture & $22.94 \pm 2.72 \mathrm{a}$ & & $18.69 \pm 3.00 \mathrm{a}$ & \\
& Substrate cultivation & $31.90 \pm 3.02 \mathrm{a}$ & 39.05 & $26.92 \pm 3.92 \mathrm{a}$ & 43.99 \\
& Hydroponics & $24.20 \pm 4.59 \mathrm{a}$ & 5.49 & $29.88 \pm 5.82 \mathrm{a}$ & 59.86 \\
\hline
\end{tabular}

Different lowercase letters within one variety represent significant difference at $P<0.05$ level

\section{Photosynthetic capacity of different tomato varieties}

Compared to $\mathrm{CK}$, the substrate cultivation and hydroponics treatment improved the net photosynthetic rate in leaves in Xinxing 101. The net photosynthetic rate in leaves in Jinbaolai, Kaidi, and Xiariyangguang were increased at seedling and mature stage as compared to CK. At flowering stage, compared with CK, the net photosynthetic rate in leaves was significantly decreased under substrate cultivation treatment, while dramatically increased under hydroponics treatment for Jinbaolai and Xiariyangguang. For Kaidi, the net photosynthetic rate in leaves at flowering stage was significantly decreased. The Pn in the varieties in response to different culture media showed similar trend at seedling stage and mature stage, while vary among culture media at flowering stage (Table 3).

The SPAD value in leaves of Xinxing101 was not affected by culture media at seedling stage and mature stage, only hydroponics treatment showed significant reduction in SPAD value at flowering stage as compared to CK. For Jinbaolai, substrate cultivation treatment induced significant reduction in SPAD value at seedling stage, while significant reduction in SPAD value at flowering stage and mature stage was detected for hydroponics treatment. Compared to $\mathrm{CK}$, the culture media (substrate cultivation and hydroponics treatment) did not affected the SPAD value in leaves at seedling stage and mature stage, while significantly decreased SPAD value in leaves at flowering stage (Table 4). 
Table 3. Effect of culture media on net photosynthetic rate in leaves of different tomato varieties $\left(\mu \mathrm{mol} \cdot \mathrm{m}^{-2} \cdot \mathrm{s}^{-1}\right)$

\begin{tabular}{c|c|c|c|c}
\hline Varieties & Culture media & Seedling stage & Flowering stage & Mature stage \\
\hline \multirow{4}{*}{ Xinxing101 } & Soil culture & $7.74 \pm 0.55 \mathrm{~b}$ & $16.01 \pm 0.07 \mathrm{~b}$ & $7.27 \pm 0.13 \mathrm{~b}$ \\
& Substrate cultivation & $9.46 \pm 0.12 \mathrm{a}$ & $18.02 \pm 0.28 \mathrm{a}$ & $8.08 \pm 0.10 \mathrm{a}$ \\
& Hydroponics & $8.64 \pm 0.30 \mathrm{ab}$ & $17.96 \pm 0.42 \mathrm{a}$ & $8.09 \pm 0.34 \mathrm{a}$ \\
\hline \multirow{3}{*}{ Jinbaolai } & Soil culture & $5.59 \pm 0.08 \mathrm{~b}$ & $14.52 \pm 0.16 \mathrm{~b}$ & $8.57 \pm 0.32 \mathrm{c}$ \\
& Substrate cultivation & $6.59 \pm 0.39 \mathrm{ab}$ & $14.06 \pm 0.11 \mathrm{c}$ & $16.34 \pm 0.35 \mathrm{a}$ \\
& Hydroponics & $7.18 \pm 0.47 \mathrm{a}$ & $18.26 \pm 0.12 \mathrm{a}$ & $10.50 \pm 0.60 \mathrm{~b}$ \\
\hline \multirow{3}{*}{ Kaidi } & Soil culture & $4.58 \pm 0.31 \mathrm{~b}$ & $19.71 \pm 0.13 \mathrm{a}$ & $11.27 \pm 0.30 \mathrm{~b}$ \\
& Substrate cultivation & $6.17 \pm 0.43 \mathrm{a}$ & $16.66 \pm 0.30 \mathrm{~b}$ & $12.24 \pm 0.16 \mathrm{~b}$ \\
& Hydroponics & $6.73 \pm 0.63 \mathrm{a}$ & $16.30 \pm 0.12 \mathrm{~b}$ & $17.56 \pm 0.85 \mathrm{a}$ \\
\hline \multirow{3}{*}{ Xiariyangguang } & Soil culture & $4.38 \pm 0.38 \mathrm{~b}$ & $14.47 \pm 0.13 \mathrm{~b}$ & $7.12 \pm 0.58 \mathrm{~b}$ \\
& Substrate cultivation & $7.31 \pm 0.53 \mathrm{a}$ & $12.58 \pm 0.08 \mathrm{c}$ & $8.33 \pm 0.33 \mathrm{~b}$ \\
& Hydroponics & $4.51 \pm 0.21 \mathrm{~b}$ & $16.10 \pm 0.14 \mathrm{a}$ & $10.41 \pm 0.62 \mathrm{a}$ \\
\hline
\end{tabular}

Different lowercase letters within one variety represent significant difference at $P<0.05$ level

Table 4. Effect of culture media on SPAD value of different tomato varieties

\begin{tabular}{c|c|c|c|c}
\hline Varieties & Culture media & Seedling stage & Flowering stage & Mature stage \\
\hline \multirow{4}{*}{ Xinxing101 } & Soil culture & $35.97 \pm 0.33 \mathrm{a}$ & $39.27 \pm 2.49 \mathrm{a}$ & $46.40 \pm 2.51 \mathrm{a}$ \\
& Substrate cultivation & $32.63 \pm 1.15 \mathrm{a}$ & $35.60 \pm 1.91 \mathrm{ab}$ & $45.30 \pm 1.07 \mathrm{a}$ \\
& Hydroponics & $35.93 \pm 1.28 \mathrm{a}$ & $32.27 \pm 0.75 \mathrm{~b}$ & $45.03 \pm 1.78 \mathrm{a}$ \\
\hline \multirow{3}{*}{ Jinbaolai } & Soil culture & $40.50 \pm 0.26 \mathrm{a}$ & $42.27 \pm 1.60 \mathrm{a}$ & $54.00 \pm 2.03 \mathrm{a}$ \\
& Substrate cultivation & $37.00 \pm 1.46 \mathrm{~b}$ & $43.20 \pm 1.44 \mathrm{a}$ & $49.60 \pm 0.78 \mathrm{ab}$ \\
& Hydroponics & $39.63 \pm 0.56 \mathrm{ab}$ & $35.83 \pm 0.80 \mathrm{~b}$ & $48.47 \pm 0.47 \mathrm{~b}$ \\
\hline \multirow{3}{*}{ Kaidi } & Soil culture & $42.93 \pm 1.87 \mathrm{a}$ & $43.20 \pm 1.44 \mathrm{a}$ & $54.67 \pm 1.33 \mathrm{a}$ \\
& Substrate cultivation & $40.00 \pm 0.45 \mathrm{a}$ & $44.17 \pm 1.11 \mathrm{a}$ & $55.43 \pm 0.97 \mathrm{a}$ \\
& Hydroponics & $42.77 \pm 1.74 \mathrm{a}$ & $39.37 \pm 1.72 \mathrm{a}$ & $51.00 \pm 0.35 \mathrm{~b}$ \\
\hline \multirow{3}{*}{ Xiariyangguang } & Soil culture & $33.57 \pm 0.58 \mathrm{ab}$ & $36.23 \pm 0.58 \mathrm{a}$ & $40.27 \pm 0.98 \mathrm{a}$ \\
& Substrate cultivation & $31.73 \pm 0.20 \mathrm{~b}$ & $32.23 \pm 0.27 \mathrm{~b}$ & $41.47 \pm 1.11 \mathrm{a}$ \\
& Hydroponics & $36.47 \pm 1.88 \mathrm{a}$ & $31.73 \pm 0.93 \mathrm{~b}$ & $40.37 \pm 1.17 \mathrm{a}$ \\
\hline
\end{tabular}

Different lowercase letters within one variety represent significant difference at $P<0.05$ level

\section{Antioxidant enzyme of different tomato varieties.}

Compared to CK, the substrate cultivation treatment significantly decreased SOD activity in leaves at mature stage for Xinxing101 and Kaidi, and at seedling stage for Xiariyangguang. Significant enhance in SOD activity in leaves at mature stage for Xiariyanguang was detected as compared to $\mathrm{CK}$. The hydroponics treatment dramatically reduced SOD activity in leaves at all the stage for Xinxing 101 and Kaidi, and at mature for Jinbaolai and Xiariyangguang as compared to CK (Table 5). 
Table 5. Effect of culture media on SOD activity in leaves of different tomato varieties $\left(U \cdot g^{-1}\right)$

\begin{tabular}{c|c|c|c|c}
\hline Varieties & Culture media & Seedling stage & Flowering stage & Mature stage \\
\hline \multirow{3}{*}{ Xinxing101 } & Soil culture & $234.94 \pm 5.54 \mathrm{a}$ & $320.16 \pm 1.39 \mathrm{a}$ & $259.34 \pm 4.70 \mathrm{a}$ \\
& Substrate cultivation & $221.56 \pm 15.81 \mathrm{a}$ & $260.07 \pm 20.41 \mathrm{ab}$ & $193.30 \pm 20.89 \mathrm{~b}$ \\
& Hydroponics & $181.57 \pm 9.69 \mathrm{~b}$ & $233.35 \pm 23.37 \mathrm{~b}$ & $166.47 \pm 16.91 \mathrm{~b}$ \\
\hline \multirow{3}{*}{ Jinbaolai } & Soil culture & $247.56 \pm 15.53 \mathrm{a}$ & $326.06 \pm 12.84 \mathrm{a}$ & $251.09 \pm 7.53 \mathrm{a}$ \\
& Substrate cultivation & $256.96 \pm 9.65 \mathrm{a}$ & $315.48 \pm 3.34 \mathrm{a}$ & $228.27 \pm 8.32 \mathrm{a}$ \\
& Hydroponics & $215.69 \pm 15.19 \mathrm{a}$ & $283.04 \pm 23.85 \mathrm{a}$ & $172.44 \pm 12.17 \mathrm{~b}$ \\
\hline \multirow{3}{*}{ Kaidi } & Soil culture & $264.07 \pm 12.57 \mathrm{a}$ & $351.54 \pm 1.51 \mathrm{a}$ & $288.39 \pm 3.48 \mathrm{a}$ \\
& Substrate cultivation & $266.10 \pm 9.43 \mathrm{a}$ & $321.86 \pm 1.98 \mathrm{ab}$ & $222.05 \pm 11.25 \mathrm{~b}$ \\
& Hydroponics & $211.29 \pm 14.88 \mathrm{~b}$ & $261.52 \pm 34.73 \mathrm{~b}$ & $203.73 \pm 19.43 \mathrm{~b}$ \\
\hline \multirow{3}{*}{ Xiariyangguang } & Soil culture & $269.83 \pm 12.01 \mathrm{a}$ & $326.51 \pm 11.83 \mathrm{a}$ & $150.64 \pm 4.88 \mathrm{~b}$ \\
& Substrate cultivation & $213.48 \pm 16.66 \mathrm{~b}$ & $304.61 \pm 2.11 \mathrm{a}$ & $193.52 \pm 0.34 \mathrm{a}$ \\
& Hydroponics & $236.79 \pm 13.09 \mathrm{ab}$ & $316.77 \pm 15.77 \mathrm{a}$ & $144.67 \pm 0.79 \mathrm{~b}$ \\
\hline
\end{tabular}

Different lowercase letters within one variety represent significant difference at $P<0.05$ level

For Xinxing 101, compared with CK, substrate cultivation treatment significantly reduced POD activity in leaves at seedling stage and mature stage, hydroponics treatment improved POD activity in leaves at seedling stage significantly, but significantly reduced POD activity in leaves at mature stage. For Jinbaolai, compared with CK, significant reduction in POD activity was investigated for substrate cultivation treatment at both seedling stage and mature stage, while hydroponics treatment significantly reduced POD activity at flowering stage and mature stage. Substrate cultivation treatment significantly reduced POD activity in leaves in Kaidi and Xiariyangguang as compared to $\mathrm{CK}$. Compared with $\mathrm{CK}$, significant reduction in POD activity in leaves at flowering stage and mature stage was recorded for hydroponics treatment (Table 6).

Table 6. Effect of culture media on POD activity in leaves of different tomato varieties $\left(U \cdot g^{-1} \cdot \mathrm{min}^{-1}\right)$

\begin{tabular}{c|c|c|c|c}
\hline Varieties & Culture media & Seedling stage & Flowering stage & Mature stage \\
\hline \multirow{3}{*}{ Xinxing101 } & Soil culture & $55.98 \pm 0.23 \mathrm{~b}$ & $382.54 \pm 69.21 \mathrm{a}$ & $640.48 \pm 94.33 \mathrm{a}$ \\
& Substrate cultivation & $38.62 \pm 4.96 \mathrm{c}$ & $235.29 \pm 43.22 \mathrm{a}$ & $412.40 \pm 51.68 \mathrm{~b}$ \\
& Hydroponics & $88.81 \pm 6.51 \mathrm{a}$ & $333.78 \pm 48.99 \mathrm{a}$ & $226.51 \pm 29.46 \mathrm{~b}$ \\
\hline \multirow{3}{*}{ Jinbaolai } & Soil culture & $71.87 \pm 7.24 \mathrm{a}$ & $278.43 \pm 9.12 \mathrm{a}$ & $407.02 \pm 15.84 \mathrm{a}$ \\
& Substrate cultivation & $9.61 \pm 0.63 \mathrm{~b}$ & $249.49 \pm 26.02 \mathrm{ab}$ & $277.01 \pm 14.67 \mathrm{~b}$ \\
& Hydroponics & $67.15 \pm 5.79 \mathrm{a}$ & $193.91 \pm 23.22 \mathrm{~b}$ & $197.78 \pm 5.94 \mathrm{c}$ \\
\hline \multirow{3}{*}{ Kaidi } & Soil culture & $87.21 \pm 3.44 \mathrm{a}$ & $242.26 \pm 16.87 \mathrm{a}$ & $391.11 \pm 34.00 \mathrm{a}$ \\
& Substrate cultivation & $22.82 \pm 1.96 \mathrm{~b}$ & $164.74 \pm 9.80 \mathrm{~b}$ & $158.47 \pm 9.08 \mathrm{~b}$ \\
& Hydroponics & $85.94 \pm 7.98 \mathrm{a}$ & $116.79 \pm 0.73 \mathrm{c}$ & $138.89 \pm 14.54 \mathrm{~b}$ \\
\hline \multirow{3}{*}{ Xiariyangguang } & Soil culture & $74.05 \pm 5.78 \mathrm{a}$ & $231.75 \pm 32.71 \mathrm{a}$ & $457.69 \pm 12.32 \mathrm{a}$ \\
& Substrate cultivation & $16.27 \pm 0.69 \mathrm{~b}$ & $95.17 \pm 12.51 \mathrm{~b}$ & $352.42 \pm 27.47 \mathrm{~b}$ \\
& Hydroponics & $85.48 \pm 3.53 \mathrm{a}$ & $87.48 \pm 3.14 \mathrm{~b}$ & $201.56 \pm 4.06 \mathrm{c}$ \\
\hline
\end{tabular}

Different lowercase letters within one variety represent significant difference at $P<0.05$ level 
Compared with CK, the substrate cultivation treatment did not affect CAT in leaves for Xinxing101, Jinbaolai, and Xiariyangguang. Significant enhancement in Kaidi at seedling stage was detected. The hydroponics treatment significantly reduced CAT activity in leaves at mature stage for Xinxing 101 and Jinbaolai as compared to CK. Compared with CK, significant improvement in CAT activity in leaves at seedling stage was investigated for Jinbaolai and Xiariyangguang, and at flowering stage for Kaidi and Xiariyangguang (Table 7).

Table 7. Effect of culture media on CAT activity in leaves of different tomato varieties $\left(U \cdot g^{-1} \cdot \mathrm{min}^{-1}\right)$

\begin{tabular}{c|c|c|c|c}
\hline Varieties & Culture media & Seedling stage & Flowering stage & Mature stage \\
\hline \multirow{3}{*}{ Xinxing101 } & Soil culture & $14.31 \pm 3.32 \mathrm{a}$ & $17.90 \pm 4.77 \mathrm{a}$ & $210.94 \pm 8.12 \mathrm{a}$ \\
& Substrate cultivation & $19.89 \pm 2.76 \mathrm{a}$ & $11.03 \pm 5.11 \mathrm{a}$ & $208.59 \pm 5.41 \mathrm{a}$ \\
& Hydroponics & $14.69 \pm 0.53 \mathrm{a}$ & $10.79 \pm 4.87 \mathrm{a}$ & $87.02 \pm 4.23 \mathrm{~b}$ \\
\hline \multirow{3}{*}{ Jinbaolai } & Soil culture & $21.57 \pm 5.04 \mathrm{~b}$ & $18.38 \pm 7.89 \mathrm{a}$ & $207.98 \pm 13.20 \mathrm{a}$ \\
& Substrate cultivation & $13.58 \pm 3.91 \mathrm{~b}$ & $14.59 \pm 6.49 \mathrm{a}$ & $216.68 \pm 2.18 \mathrm{a}$ \\
& Hydroponics & $39.39 \pm 4.83 \mathrm{a}$ & $4.82 \pm 0.04 \mathrm{a}$ & $80.10 \pm 12.42 \mathrm{~b}$ \\
\hline \multirow{3}{*}{ Kaidi } & Soil culture & $7.61 \pm 0.64 \mathrm{~b}$ & $9.68 \pm 2.37 \mathrm{~b}$ & $152.13 \pm 29.35 \mathrm{ab}$ \\
& Substrate cultivation & $42.01 \pm 8.54 \mathrm{a}$ & $17.50 \pm 4.82 \mathrm{~b}$ & $198.67 \pm 49.19 \mathrm{a}$ \\
& Hydroponics & $13.51 \pm 3.66 \mathrm{~b}$ & $31.09 \pm 2.65 \mathrm{a}$ & $77.34 \pm 1.35 \mathrm{~b}$ \\
\hline \multirow{3}{*}{ Xiariyangguang } & Soil culture & $8.62 \pm 0.00 \mathrm{~b}$ & $13.35 \pm 2.18 \mathrm{~b}$ & $126.03 \pm 3.28 \mathrm{a}$ \\
& Substrate cultivation & $15.79 \pm 0.00 \mathrm{a}$ & $19.25 \pm 1.44 \mathrm{ab}$ & $140.52 \pm 9.41 \mathrm{a}$ \\
& Hydroponics & $15.07 \pm 1.22 \mathrm{a}$ & $21.74 \pm 2.80 \mathrm{a}$ & $139.23 \pm 2.16 \mathrm{a}$ \\
\hline
\end{tabular}

Different lowercase letters within one variety represent significant difference at $P<0.05$ level

\section{Correlation analysis}

Significant correlation relationship between yield and yield per plant and fruit weight $\left(\mathrm{R}^{2}=0.8205, P<0.01\right)$, net photosynthetic rate in leaves at seedling stage $\left(\mathrm{R}^{2}=0.7189\right.$, $P<0.05$ ) was detected (Fig. 1).


Figure 1. Correlation relationship between yield per plant and fruit weight, net photosynthetic rate in leaves at seedling stage. $* *$ and $*$ represent significant difference at $P<0.01$ and $P<0.05$ level, respectively 


\section{Discussion}

Soilless culture mainly includes water culture, fog culture and substrate culture, among which substrate culture is the most important form of soilless culture. The main function of culture media is to support and fix plants, and provide a stable and coordinated water, gas and fertilizer environment for the crops (Liu et al., 2006). In the traditional sense, soilless cultivation mainly relies on nutrient solution to supply the total value nutrients needed by crops, and the nutrient content of cultivation matrix is often ignored whilst in fact, various organic substrates are rich in nutrients. In this study, we observed higher yield was recorded in both substrate cultivation and hydroponics than soil culture. This result agreed with the study of Tzortzakis (Tzortzakis and Economakis, 2008) which found that tomato grown in special substrate had higher yield and better fruit quality. There are several factors which effected yield and photosynthesis. The researches of Zhu (Zhu et al., 2012) showed that the interaction of water and fertilizer affected photosynthetic capacity and yield significantly in tomato plants. And in our study, the substrate cultivation and hydroponics indeed had some differences in net photosynthetic rate compared with soil culture. Meanwhile, early study proved that the net photosynthetic rate was a limited factor for yield, dry matter and fruit quality (Whitehead and Singh, 1997). Our research evidenced the view again by showing a significant positive correlation between yield and net photosynthetic rate in leaves at seedling stage.

In addition, we also found that the hydroponics treatment and substrate cultivation treatment reduced the activity of SOD or POD in leaves for some tomato cultivars in some stage. SOD and POD anti-oxidative enzymatic which have significant roles in osmo-regulations and often help to quench ROS while maintaining cellular structures and functions (Kong et al., 2017). For example, SOD dismutases superoxide radical when POD involved in scavenging $\mathrm{H}_{2} \mathrm{O}_{2}$ (Sairam et al., 2000). The changes in activity of SOD and POD might suggest that the environment of substrate cultivation and hydroponics was more stable and less environmental stresses for tomato and thus the tomato could had higher dry weight accumulation, fruit weight, and net photosynthetic rate.

In recent years, with the development of ecological agriculture and sustainable circular agriculture, greenhouse tomato in media culture develops rapidly. We would suggest that the substrate cultivation and hydroponics is benefit for tomato growth because they produced higher yield as a consequences of higher dry weight accumulation, fruit weight, and net photosynthetic rate at seedling stage and mature stage.

\section{Conclusion}

The substrate cultivation and hydroponics produced higher yield than soil culture treatment due to higher dry weight accumulation, fruit weight, and net photosynthetic rate at seedling stage and mature stage. The yield showed significant associate to fruit weight $\left(\mathrm{R}^{2}=0.8205, P<0.01\right)$, net photosynthetic rate in leaves at seedling stage $\left(\mathrm{R}^{2}=0.7189, P<0.05\right)$. The SPAD value and antienzyme activity vary from different planting patterns, cultivars and growth stages.

Acknowledgements. This research was supported by the Project of Display of Super Vegetable Cultivation Mode in National Agricultural Science and Technology Park, Guangzhou Guangdong province (2014 A070709007), Display of Special Rice and Vegetable Germplasm Collection in National Agricultural Science and Technology Park, Guangzhou Guangdong province (2015A070708010). 
Author contributions. Liangliang Shi, Jinrong Zheng and Jianwu Wang designed the research, Liangliang Shi, Na Lv, Delong Tan and Jun Nie performed the experiments and collected the data, Liangliang Shi and $\mathrm{Na} \mathrm{Lv}$ analyzed the data and wrote the manuscript, Guoqing Luo, Yuming Xie, Yanhong Li and Yan Xiong edited the manuscript and provided guidance during experimentation.

\section{REFERENCES}

[1] Abou-Hadid, A. F., El-Shinawy, M. Z., El-Oksh, I., Gomaa, H., El-Beltagy, A. S. (1993): Studies on water consumption of sweet pepper plant under plastic houses. - II Symposium on Protected Cultivation of Solanacea in Mild Winter Climates 366: 365372.

[2] Chen, S., Cheng, W., Liu, A., He, C., Zou, Z. (2009): Effect of different organic substrate on leaf photosynthesis and root ATPase activity of tomato. - China Vegetables (14): 2327.

[3] Foyer, C. H. (1987): The basis for source-sink interaction in leaves. - Plant Physiol Biochem 25(5): 649-657.

[4] Ghoreishy, F., Ghehsareh, A. M., Fallahzade, J. (2018): Using composted wheat residue as a growth medium in culture of tomato. - Journal of Plant Nutrition 41(6): 766-773.

[5] Khan, F. A. A. (2018): A Review on hydroponic greenhouse cultivation for sustainable agriculture. - International Journal of Agriculture, Environment and Food Sciences 2(2): 59-66.

[6] Kong, L., Ashraf, U., Cheng, S., Rao, G., Mo, Z., Tian, H., Pan, S., Tang, X. (2017): Short-term water management at early filling stage improves early-season rice performance under high temperature stress in South China. - European Journal of Agronomy 90: 117-126.

[7] Korkmaz, A., Karagöl, A., Horuz, A. (2016): Effect of NaCI in solid media culture on the yield and fruit quality of tomato plant. - Anadolu Tarım Bilimleri Dergisi 31(1): 127-135.

[8] Lee, J. W., Kim, Y. C., Yun, H. K., Seo, T. C., Park, K. W. (2002): Influence of silicate application on the sucrose synthetic enzyme activity of tomato in perlite media culture. XXVI International Horticultural Congress: Protected Cultivation 2002: In Search of Structures, Systems and Plant Materials for for Sustainable Greenhouse Production. ISHS Acta Horticulturae 633: 259-262.

[9] Liu, W., Yu, H. J., Jiang, W. J. (2006): Review on research progress and application of growing media for vegetable production in China. - Chinese Journal of Eco-Agriculture 14(3): 4-7.

[10] Lv, J., Sang, P., Li, L., Li, X. (2010): Effect of nutrient solution with different formulas and concentrations on the growth of tomato seedling. - Journal of Shanxi Agricultural University (Natural Science Edition) 2: 8.

[11] Mehdizadeh, M., Darbandi, E. I., Naseri-Rad, H., Tobeh, A. (2013): Growth and yield of tomato (Lycopersicon esculentum Mill.) as influenced by different organic fertilizers. International Journal of Agronomy and Plant Production 4(4): 734-738.

[12] Pires, R. C. D. M., Furlani, P. R., Ribeiro, R. V., Bodine Junior, D., Sakai, E., Lourenção, A. L., Torre Neto, A. (2011): Irrigation frequency and substrate volume effects in the growth and yield of tomato plants under greenhouse conditions. - Scientia Agricola 68(4): 400-405.

[13] Sairam, R. K., Srivastava, G. C., Saxena, D. C. (2000): Increased antioxidant activity under elevated temperatures: a mechanism of heat stress tolerance in wheat genotypes. Biologia Plantarum 43(2): 245-251.

[14] Salokhe, V. M., Babel, M. S., Tantau, H. J. (2005): Water requirement of drip irrigated tomatoes grown in greenhouse in tropical environment. - Agricultural Water Management 71(3): 225-242. 
[15] Tichá, I., Čáp, F., Pacovská, D., Hofman, P., Haisel, D., Čapková, V., Schäfer, C. (1998): Culture on sugar medium enhances photosynthetic capacity and high light resistance of plantlets grown in vitro. - Physiologia Plantarum 102(2): 155-162.

[16] Tzortzakis, N. G., Economakis, C. D. (2008): Impacts of the substrate medium on tomato yield and fruit quality in soilless cultivation. - Hort. Sci 35(2): 83-89.

[17] Whitehead, W. F., Singh, B. P. (1997): Effect of N source on photosynthesis and plant dry matter yield of tomato. - HortScience 32(3): 518-518.

[18] Xie, J., Yu, J., Chen, B., Feng, Z., Li, J., Zhao, C., Lyu, J., Hu, L., Gan, Y., Siddique, K. (2017): Facility cultivation systems “设施农业”: a Chinese model for the Planet. Advances in Agronomy 145: 1-42.

[19] Xu, H., Wang, R., Mau, M. (2001): Effects of organic fertilizers and a microbial inoculant on leaf photosynthesis and fruit yield and quality of tomato plants. - Journal of Crop Production 1: 173-182.

[20] Yang, Y., Zhang, F., Qiao, X. (2003): Effect of nitrogen forms on growth development, yield and fruit quality of tomato in media culture. - Acta Agriculturae Boreali Sinica 18(1): 86-89.

[21] Zhu, J., Liang, Y., Zhu, Y., Hao, W., Lin, X., Xing, W., Luo, A. (2012): The interactive effects of water and fertilizer on photosynthetic capacity and yield in tomato plants. Australian Journal of Crop Science 2: 200-209. 